\title{
Neutrophil-lymphocyte ratio: Can clinicians really trust it as an inflammatory indicator?
}

We have read the article 'Neutrophil to lymphocyte ratio (NLR) predicts short- and long-term mortality following revascularization therapy for ST elevation myocardial infarction (STEMI)' by Sawant et al. [1]. The authors investigated if NLR, as an all-cause mortality outcome predictor, could be utilized alone, and in combination with current standardized cardiac risk score models, improved the prediction of subsequent short and long-term all-cause mortality. They concluded that the NLR based on an optimal cut-off value of 7.4 was an excellent predictor of short- and long-term survival in patients with revascularized STEMI and warrants larger scale multi-center prospective evaluation, as a prognostic indicator. NLR offers improved prognostic capacity when combined with conventional clinical scoring systems, such as the TIMI risk score. We would like to thank to the authors for their contribution.

The pathogenesis of atherosclerosis is multifactorial; however, it is mostly considered an inflammatory condition [2]. Atherosclerotic diseases, especially STEMI, are of high mortality and morbidity rates in the world. Previous data demonstrated that elevated levels of inflammatory molecules indicate an increased risk of the progression of atherosclerosis [3]. The complete blood count $(\mathrm{CBC})$ is one of the most frequently ordered laboratory tests in clinical practice. Various studies have evaluated the performance of these hematological $\mathrm{CBC}$ parameters to predict disease severity and mortality risk. White blood cell count and its subtypes including NLR can be easily used as inflammatory biomarkers in clinical practice $[3,4]$. We consider the associations between the NLR and a vascular disease and its associated risk factors. The NLR can be affected by atherosclerotic risk factors, such as hypercholesterolemia, metabolic syndrome, diabetes, and hypertension. Importantly, it can predict mortality in cardiovascular diseases. There are also reports of a positive correlation between the NLR and commonly used inflammatory markers. In addition, inflammatory diseases like Behçet's disease (BD) and psoriasis are associated with inflammatory markers [5]. In context, the NLR may use activity marker for BD patients. We have shown that NLR levels were significantly higher in active $\mathrm{BD}$ patients compared with inactive patients [6].

In conclusion, NLR is an easy method to assess inflammation and it may be useful in prognostic indicator of many conditions. However, NLR alone, without other inflammatory markers, may not give information to clinicians about the short- or long-term survival in patients with revascularized STEMI. Therefore, we think that it should be evaluated together with other serum inflammatory markers.

\section{Conflict of interest: None declared}

\section{References}

1. Sawant AC, Adhikari P, Narra SR, Srivatsa SS, Mills PK, Srivatsa SS. Neutrophil to lymphocyte ratio predicts short- and long-term mortality following revascularization therapy for ST elevation myocardial infarction. Cardiol J, 2014; 21: 500-508. doi: 10.5603/ CJ.a2013.0148.

2. Balta S, Demirkol S, Kucuk U, Celik T, Ozturk C, Iyisoy A. The relationship between neutrophil-lymphocyte ratio and coronary collateral circulation. Perfusion, 2014; 29: 367-368.

3. Balta S, Celik T, Mikhailidis DP et al. The relation between atherosclerosis and the neutrophil-lymphocyte ratio. Clin Appl Thromb Hemost, 2015 Feb 9, pii: 1076029615569568 [Epub ahead of print].

4. Demirkol S, Balta S, Cakar M, Unlu M, Arslan Z, Kucuk U. Red cell distribution width: a novel inflammatory marker in clinical practice. Cardiol J, 2013; 20: 209. doi: 10.5603/CJ.2013.0037.

5. Balta S, Balta I, Mikhailidis DP et al. Bilirubin levels and their association with carotid intima media thickness and high-sensitivity C-reactive protein in patients with psoriasis vulgaris. Am J Clin Dermatol, 2014; 15: 137-142. doi: 10.1007/s40257-014-0069-5.

6. Ozturk C, Balta S, Balta I et al. Neutrophil-lymphocyte ratio and carotid-intima media thickness in patients with behcet disease without cardiovascular involvement. Angiology, 2015; 66: 291-296. doi: 10.1177/0003319714527638.

Sevket Balta, Sait Demırkol, Murat Unlu, Cengiz Ozturk, Zekeriya Arslan, Turgay Celık Department of Cardiology, Gulhane School of Medicine, Tevfik Saglam St. 06018 Etlik-Ankara, Turkey tel: +9031230442 81, fax: +903123044250 e-mail:drsevketb@gmail.com;sevketb@gata.edu.tr 